\title{
An Efficient Higher-Order Quasilinearization Method for Solving Nonlinear BVPs
}

\author{
Eman S. Alaidarous, ${ }^{1}$ Malik Zaka Ullah,, ${ }^{1}$ Fayyaz Ahmad, ${ }^{2}$ and A.S. Al-Fhaid ${ }^{1}$ \\ ${ }^{1}$ Department of Mathematics, King Abdulaziz University, Jeddah 21589, Saudi Arabia \\ ${ }^{2}$ Departamento de Física i Enginyeria Nuclear, Universitat Politècnica de Catalunya, Compte d'Urgell 187, 08036 Barcelona, Spain \\ Correspondence should be addressed to Fayyaz Ahmad; fayyaz.ahmad@upc.edu
}

Received 28 August 2013; Accepted 22 September 2013

Academic Editor: K. S. Govinder

Copyright (c) 2013 Eman S. Alaidarous et al. This is an open access article distributed under the Creative Commons Attribution License, which permits unrestricted use, distribution, and reproduction in any medium, provided the original work is properly cited.

In this research paper, we present higher-order quasilinearization methods for the boundary value problems as well as coupled boundary value problems. The construction of higher-order convergent methods depends on a decomposition method which is different from Adomain decomposition method (Motsa and Sibanda, 2013). The reported method is very general and can be extended to desired order of convergence for highly nonlinear differential equations and also computationally superior to proposed iterative method based on Adomain decomposition because our proposed iterative scheme avoids the calculations of Adomain polynomials and achieves the same computational order of convergence as authors have claimed in Motsa and Sibanda, 2013. In order to check the validity and computational performance, the constructed iterative schemes are also successfully applied to bifurcation problems to calculate the values of critical parameters. The numerical performance is also tested for one-dimension Bratu and Frank-Kamenetzkii equations.

\section{Introduction}

In the very beginning, the Newton-Raphson method constructed for nonlinear single-variable algebraic equation whose efficiency index is optimal according to Kung-Traub conjecture [1], actually Newton-Raphson uses two function evaluations, and its computational order of convergence is two. Many authors [2-7] have made good effort to construct the iterative methods for algebraic equations. Similarly, for the system of algebraic equation, the version of NewtonRaphson gives us second-order convergence. In the literature, the efficiency index is only defined for single-variable algebraic equation on the basis of function evaluations. For multivariable case, if we consider system of $n$ algebraic equations, then we require the computation of Jacobian inverse $J^{-1}$ of dimension $n \times n$ and $n$ function evaluations to perform one newton iteration and it is clearly evident the computational cost of matrix inversion is dominant over other binary operations to complete one newton iteration. The iterative methods which require only once the Jacobian inverse for the whole cycle of iterations are clearly efficient.
When we talk about nonlinear boundary value problems, the quasilinear method (QLM) [8-11] is an iterative method which starts from initial guess for a boundary value problem (BVP) which is quadratically convergent. Initially, Bellman and Kalaba [12] proposed QLM and later Mandelzweig and coauthors [8-11] provide the second-order convergence proof for the BVPs. In [13], recently authors proposed higher-order quasilinearization method for single BVP as well as coupled BVPs. The original idea in [13] is to decompose the nonlinear operator as an infinite sum of Adomain [14] polynomials. The reported algorithm is efficient in the case of couple BVPs. The computation of Jacobian inverse is performed at the initial guess, but the calculation of Adomain polynomials is somehow difficult and also increases the computation cost of iterative scheme. To avoid the computational burden of Adomain polynomials, we use a different decomposition method for nonlinear operator which was actually introduced in [15]. Our proposed scheme uses only one calculation of Jacobian inverse $J^{-1}$ and does not require any calculation of Adomain polynomials, and this fact increases its computational efficiency in comparison with [13]. The sequences of 
iteration schemes have convergence orders two, three, four, five, and so forth. The numerical stability and efficiency are tested over two problems, namely, one-dimensional Bratu problem [16-23]

$$
y^{\prime \prime}(x)+\lambda e^{y(x)}=0, \quad y(0)=y(1)=0
$$

and Frank-Kamenetzkii [24] boundary value problem

$$
y^{\prime \prime}(x)+\frac{k}{x} y^{\prime}(x)+\lambda e^{y(x)}=0, \quad y^{\prime}(0)=y(1)=0
$$

The Frank-Kamenetzkii BVP (2) has no solution if $\lambda>2$, unique solution if $\lambda=2$ and two solutions if $\lambda<2$. The closed form solution of (2) is reported in [24-26]. The solutions of (2) in [25] are given as

$$
\begin{gathered}
c_{1}=\log [2(4-\lambda) \pm 4 \sqrt{2(2-\lambda)}], \\
c_{2}=\log \left[\frac{4-\lambda \pm 2 \sqrt{2(2-\lambda)}}{2 \lambda^{2}}\right], \\
y=\log \left[\frac{16 e_{1}^{c}}{\left(2 \lambda+e^{c_{1}} x^{2}\right)^{2}}\right], \quad y=\log \left[\frac{16 e_{1}^{c}}{\left(1+2 \lambda e^{c_{2}} x^{2}\right)^{2}}\right] .
\end{gathered}
$$

The closed form solution for Bratu equation [27] can be written as

$$
\begin{gathered}
y(x)=-2 \log \left[\frac{\cosh ((x-1 / 2)(\theta / 2))}{\cosh (\theta / 4)}\right] \\
\theta=\sqrt{2 \lambda} \cosh \left(\frac{\theta}{4}\right) .
\end{gathered}
$$

The critical parameter $\lambda_{c}$ for Bratu problem satisfies $4=$ $\sqrt{2 \lambda_{c}} \sinh \left(\theta_{c} / 4\right)$ and if $\lambda<\lambda_{c}, \lambda=\lambda_{c}$, and $\lambda>\lambda_{c}$, then there are two solutions, unique solution and no solution for (1). The numerical reported value of critical parameter is $\lambda_{c}=$ 3.51383071912516 [18].

\section{Construction of Iterative Methods}

2.1. Single Nonlinear Boundary Value Problem. Consider a nonlinear ordinary differential equation

$$
L(y)+f(y)=0
$$

where $L$ is a linear derivative operator; for Bratu and FrankKamenetzkii problems linear operators are $L[y]=d^{2} y / d x^{2}$ and $L[y]=d^{2} y / d x^{2}+(k / x)(d y / d x)$, and $f$ is any nonlinear function of $y$. Let $\gamma$ be an initial guess (satisfying the boundary conditions) for the solution $y$ of (5). By expanding $f$ around $\gamma$, we obtain

$$
\begin{gathered}
f(y-\gamma+\gamma)=f(\gamma)+(y-\gamma) f_{y}(\gamma)+g(y), \\
g(y)=f(y)-f(\gamma)-(y-\gamma) f_{y}(\gamma), \\
\text { where } f_{y}(\gamma)=\left.\frac{\partial f(y)}{\partial y}\right|_{y=\gamma},
\end{gathered}
$$

$$
M(y)=y-\frac{f(y)}{f_{y}(\gamma)}-\gamma+\frac{f(\gamma)}{f_{y}(\gamma)},
$$

$$
\text { where } M(y)=-\frac{g(y)}{f_{y}(\gamma)},
$$

$$
\begin{gathered}
M(y)+c=y-\frac{f(y)}{f_{y}(\gamma)}, \quad \text { where } c=\gamma-\frac{f(\gamma)}{f_{y}(\gamma)}, \\
L(y)+f(\gamma)+(y-\gamma) f_{y}(\gamma)+g(y)=0, \\
\frac{L(y)}{f_{y}(\gamma)}+\frac{f(\gamma)}{f_{y}(\gamma)}+(y-\gamma)=M(y), \\
\frac{L(y)}{f_{y}(\gamma)}+y=c+M(y) .
\end{gathered}
$$

Suppose, that we can decompose the solution $y$ into infinite series sum

$$
y=\sum_{i=0}^{\infty} y_{i}
$$

Further, we obtain the decomposition of nonlinear operator $M(y)$ as follows:

$$
\begin{aligned}
M(y)= & M\left(\sum_{i=0}^{\infty} y_{i}\right)=M\left(y_{0}\right)+\left(M\left(y_{0}+y_{1}\right)-M\left(y_{0}\right)\right) \\
& +\left(M\left(y_{0}+y_{1}+y_{2}\right)-M\left(y_{0}+y_{1}\right)\right)+\cdots \\
M\left(y_{0}+\right. & \left.y_{1}+\cdots+y_{n+1}\right) \\
= & M\left(y_{0}\right)+\left(M\left(y_{0}+y_{1}\right)-M\left(y_{0}\right)\right) \\
& +\left(M\left(y_{0}+y_{1}+y_{2}\right)-M\left(y_{0}+y_{1}\right)\right)+\cdots \\
& +\left(M\left(y_{0}+y_{1}+\cdots+y_{n+1}\right)\right. \\
& \left.\quad-M\left(y_{0}+y_{1}+\cdots+y_{n}\right)\right)
\end{aligned}
$$

By substituting (11) in (10), we get

$$
\begin{aligned}
& \frac{L\left(y_{0}+y_{1}+y_{2}+\cdots\right)}{f_{y}(\gamma)}+\left(y_{0}+y_{1}+y_{2}+\cdots\right) \\
& =c+M\left(\sum_{i=0}^{\infty} y_{i}\right) .
\end{aligned}
$$


By using (13), we obtain

$$
\begin{aligned}
& \frac{L\left(y_{0}+y_{1}+y_{2}+\cdots\right)}{f_{y}(\gamma)}+\left(y_{0}+y_{1}+y_{2}+\cdots\right) \\
& =c+M\left(y_{0}\right)+\left(M\left(y_{0}+y_{1}\right)-M\left(y_{0}\right)\right)+\cdots .
\end{aligned}
$$

By comparing left and right sides in (15), we have

$$
\begin{gathered}
\frac{L\left(y_{0}\right)}{f_{y}(\gamma)}+y_{0}=c, \\
\frac{L\left(y_{1}\right)}{f_{y}(\gamma)}+y_{1}=M\left(y_{0}\right), \\
\frac{L\left(y_{2}\right)}{f_{y}(\gamma)}+y_{2}=M\left(y_{0}+y_{1}\right)-M\left(y_{0}\right) \\
\vdots \\
\frac{L\left(y_{n+1}\right)}{f_{y}(\gamma)}+y_{n+1}=M\left(y_{0}+y_{1}+\cdots+y_{n}\right) \\
-M\left(y_{0}+y_{1}+\cdots+y_{n-1}\right) .
\end{gathered}
$$

If we approximate solution

$$
y \approx Y_{n+1}=y_{0}+y_{1}+\cdots+y_{n+1}
$$

By adding (16) to (18), we get

$$
\begin{gathered}
\frac{L\left(y_{0}+y_{1}+\cdots+y_{n+1}\right)}{f_{y}(\gamma)}+y_{0}+y_{1}+\cdots+y_{n+1} \\
=c+M\left(y_{0}+y_{1}+\cdots+y_{n}\right), \\
\frac{L\left(y_{0}+y_{1}+\cdots+y_{n+1}\right)}{f_{y}(\gamma)}+y_{0}+y_{1}+\cdots+y_{n+1} \\
=y_{0}+y_{1}+\cdots+y_{n}-\frac{f\left(y_{0}+y_{1}+\cdots+y_{n}\right)}{f_{y}(\gamma)}, \\
L\left(Y_{n+1}\right)+f_{y}(\gamma) Y_{n+1}=f_{y}(\gamma) Y_{n}-f\left(Y_{n}\right) .
\end{gathered}
$$

Equations (16) and (21) give

$$
\begin{gathered}
L\left(Y_{0}\right)+Y_{0}=f_{y}(\gamma) \gamma-f(\gamma), \quad \text { where } Y_{0}=y_{0}, \\
L\left(Y_{n+1}\right)+f_{y}(\gamma) Y_{n+1}=f_{y}(\gamma) Y_{n}-f\left(Y_{n}\right), \quad n=0,1, \ldots
\end{gathered}
$$

After renaming the variables, we obtain the following iterative schemes.

Scheme $S_{0}$. Consider

$$
L\left(y_{1}\right)+f_{y}\left(y_{0}\right) y_{1}=f_{y}\left(y_{0}\right) y_{0}-f\left(y_{0}\right) .
$$

Note that $S_{0}$ corresponds to the QLM scheme which is quadratically convergent.

Scheme $S_{1}$. Consider

$$
\begin{aligned}
& L\left(y_{1}\right)+f_{y}\left(y_{0}\right) y_{1}=f_{y}\left(y_{0}\right) y_{0}-f\left(y_{0}\right), \\
& L\left(y_{2}\right)+f_{y}\left(y_{0}\right) y_{2}=f_{y}\left(y_{0}\right) y_{1}-f\left(y_{1}\right) .
\end{aligned}
$$

Scheme $S_{2}$. Consider

$$
\begin{aligned}
& L\left(y_{1}\right)+f_{y}\left(y_{0}\right) y_{1}=f_{y}\left(y_{0}\right) y_{0}-f\left(y_{0}\right), \\
& L\left(y_{2}\right)+f_{y}\left(y_{0}\right) y_{2}=f_{y}\left(y_{0}\right) y_{1}-f\left(y_{1}\right), \\
& L\left(y_{3}\right)+f_{y}\left(y_{0}\right) y_{3}=f_{y}\left(y_{0}\right) y_{2}-f\left(y_{2}\right) .
\end{aligned}
$$

Scheme $S_{n}$. Consider

$$
\begin{aligned}
& L\left(y_{1}\right)+f_{y}\left(y_{0}\right) y_{1}=f_{y}\left(y_{0}\right) y_{0}-f\left(y_{0}\right), \\
& L\left(y_{2}\right)+f_{y}\left(y_{0}\right) y_{2}=f_{y}\left(y_{0}\right) y_{1}-f\left(y_{1}\right), \\
& L\left(y_{3}\right)+f_{y}\left(y_{0}\right) y_{3}=f_{y}\left(y_{0}\right) y_{2}-f\left(y_{2}\right),
\end{aligned}
$$

$$
L\left(y_{n+1}\right)+f_{y}\left(y_{0}\right) y_{n+1}=f_{y}\left(y_{0}\right) y_{n}-f\left(y_{n}\right) .
$$

By calculating computational order of convergence, we show that the order of convergence of $S_{n}$ is $n+2$.

Consider the following nonlinear coupled boundary value problem:

$$
\begin{aligned}
& L_{1}\left(z^{(1)}\right)+f_{1}(z)=0, \\
& L_{2}\left(z^{(2)}\right)+f_{2}(z)=0, \\
& L_{3}\left(z^{(3)}\right)+f_{3}(z)=0,
\end{aligned}
$$

$$
L_{n}\left(z^{(n)}\right)+f_{n}(z)=0
$$

where $z=\left[z^{(1)}, z^{(2)}, z^{(3)}, \ldots, z^{(n)}\right]^{T}$ and $L_{1}, L_{2}, L_{3}, \ldots, L_{n}$ are linear derivative operators. Equation (27) can be rewritten as

$$
L(z)+f(z)=0,
$$

where $L=\left[L_{1}, L_{2}, L_{3}, \ldots, L_{n}\right]^{T}$ and $f(z)=\left[f_{1}(z), f_{2}(z)\right.$, $\left.\ldots, f_{n}(z)\right]^{T}$. Let $\gamma=\left[\gamma_{1}, \gamma_{2}, \ldots, \gamma_{n}\right]$ be an initial guess, which 
satisfies boundary conditions, for problem (28). Taylor's expansion of $f$ around $\gamma$ is

$$
\begin{gathered}
f(z)=f(\gamma)+J(z-\gamma)+g(z), \\
\text { where } J \text { is a Jacobian } J=\left.\frac{\partial f}{\partial z}\right|_{z=\gamma}, \\
g(z)=f(z)-f(\gamma)-J(z-\gamma), \\
-J^{-1} g(z)=-J^{-1} f(z)+J^{-1} f(\gamma)+z-\gamma, \\
M(z)+c=z-J^{-1} f(z), \quad \text { where } c=\gamma-J^{-1} f(\gamma), \\
M(z)=-J^{-1} g(z) .
\end{gathered}
$$

Equation (28) can be written as

$$
\begin{aligned}
J^{-1} L(z)+J^{-1} f(z) & =0, \quad(30) \text { implies } \\
J^{-1} L(z)+z & =M(z)+c,
\end{aligned}
$$

where $z$ can be decomposed into infinite series sum

$$
z=\sum_{i=0}^{\infty} z_{i}
$$

Substituting (33) in (32), we get

$$
J^{-1} L\left(\sum_{i=0}^{\infty} z_{i}\right)+\sum_{i=0}^{\infty} z_{i}=M\left(\sum_{i=0}^{\infty} z_{i}\right)+c .
$$

By using the same decomposition for nonlinear operator $M(z)$ for multivariable case which is given in (12), we obtain

$$
\begin{gathered}
J^{-1} L\left(z_{0}\right)+z_{0}=c, \\
J^{-1} L\left(z_{1}\right)+z_{1}=M\left(z_{0}\right), \\
J^{-1} L\left(z_{2}\right)+z_{2}=M\left(z_{0}+z_{1}\right)-M\left(z_{0}\right), \\
J^{-1} L\left(z_{3}\right)+z_{3}=M\left(z_{0}+z_{1}+z_{2}\right)-M\left(z_{0}+z_{1}\right), \\
\vdots \\
J^{-1} L\left(z_{n+1}\right)+z_{n+1}=M\left(z_{0}+z_{1}+\cdots+z_{n}\right) \\
-M\left(z_{0}+z_{1}+\cdots+z_{n-1}\right) .
\end{gathered}
$$

If we approximate the solution by

$$
z \approx Z_{n+1}=z_{0}+z_{1}+\cdots+z_{n+1},
$$

we get by adding (35)

$$
J^{-1} L\left(Z_{n+1}\right)+Z_{n+1}=c+M\left(Z_{n}\right) .
$$

From (32), we have

$$
\begin{gathered}
J^{-1} L\left(Z_{n+1}\right)+Z_{n+1}=Z_{n}-J^{-1} f\left(Z_{n}\right), \\
L\left(Z_{n+1}\right)+J Z_{n+1}=J Z_{n}-f\left(Z_{n}\right) .
\end{gathered}
$$

After renaming the variables, we get the following iterative schemes.

Scheme $S M_{0}$. Consider

$$
L\left(z_{1}\right)+J z_{1}=J z_{0}-f\left(z_{0}\right), \quad \text { where } J=\left.\frac{\partial f}{\partial z}\right|_{z=z_{0}} .
$$

Note that $S M_{0}$ is the QLM scheme which is quadratically convergent.

Scheme $S M_{1}$. Consider

$$
\begin{aligned}
& L\left(z_{1}\right)+J z_{1}=J z_{0}-f\left(z_{0}\right), \\
& L\left(z_{2}\right)+J z_{2}=J z_{1}-f\left(z_{1}\right) .
\end{aligned}
$$

Scheme $S M_{2}$. Consider

$$
\begin{aligned}
& L\left(z_{1}\right)+J z_{1}=J z_{0}-f\left(z_{0}\right), \\
& L\left(z_{2}\right)+J z_{2}=J z_{1}-f\left(z_{1}\right), \\
& L\left(z_{3}\right)+J z_{3}=J z_{2}-f\left(z_{2}\right) .
\end{aligned}
$$

Scheme $S M_{n}$. Consider

$$
\begin{aligned}
& L\left(z_{1}\right)+J z_{1}=J z_{0}-f\left(z_{0}\right), \\
& L\left(z_{2}\right)+J z_{2}=J z_{1}-f\left(z_{1}\right), \\
& L\left(z_{3}\right)+J z_{3}=J z_{2}-f\left(z_{2}\right),
\end{aligned}
$$

$$
L\left(z_{n+1}\right)+J z_{n+1}=J z_{n}-f\left(z_{n}\right) .
$$

2.2. Coupled Boundary Value with Many Variables. Consider the following nonlinear coupled boundary value problem with many variables:

$$
\begin{gathered}
L_{1}\left(z^{(1)}\right)+f_{1}(w)=0, \\
L_{2}\left(z^{(2)}\right)+f_{2}(w)=0, \\
L_{3}\left(z^{(3)}\right)+f_{3}(w)=0, \\
\vdots \\
L_{n}\left(z^{(n)}\right)+f_{n}(w)=0,
\end{gathered}
$$

where $w=\left[z^{(1)}, z^{(2)}, z^{(3)}, \ldots, z^{(n)}, s^{(1)}, s^{(2)}, \ldots, s^{(m)}\right]^{T}$. The compact form of (43) is

$$
L(z)+f(w)=0,
$$

where $z=\left[z^{(1)}, z^{(2)}, z^{(3)}, \ldots, z^{(n)}\right]^{T}, s=\left[s^{(1)}, s^{(2)}, \ldots, s^{(m)}\right]^{T}$, $L=\left[L_{1}\left(z^{(1)}\right), L_{2}\left(z^{(2)}\right), \ldots, L_{n}\left(z^{(n)}\right)\right]^{T}, f(w)=\left[f_{1}(w), f_{2}(w)\right.$, $\left.\ldots, f_{n}(w)\right]^{T}$. Let $\delta=\left[\gamma^{T}, \eta^{T}\right]^{T}$ be an initial guess for (44) which satisfies the boundary conditions where 
$\gamma=\left[\gamma_{1}, \gamma_{2}, \ldots, \gamma_{n}\right]^{T}$ and $\eta=\left[\eta_{1}, \eta_{2}, \ldots, \eta_{m}\right]^{T}$. The expansion of $f$ around $\delta$ is

$$
\begin{gathered}
f(w)=f(\delta)+J(w-\delta)+g(w), \\
f(w)=f(\delta)+J_{1}(z-\gamma)+J_{2}(s-\eta)+g(w), \\
\text { where } J_{1}=\left.\frac{\partial f}{\partial z}\right|_{z=\gamma}, J_{2}=\left.\frac{\partial f}{\partial s}\right|_{s=\eta}, \\
J_{1}^{-1} f(w)=J_{1}^{-1} f(\delta)+z-\gamma+J_{1}^{-1} J_{2}(s-\eta)+J_{1}^{-1} g(w), \\
M(w)=-J_{1}^{-1} f(w)+J_{1}^{-1} f(\delta)+z-\gamma+J_{1}^{-1} J_{2}(s-\eta), \\
\text { where } M(w)=-J_{1}^{-1} g(w), \\
M(w)+\gamma+J_{1}^{-1} J_{2} \eta-J_{1}^{-1} f(\delta)=-J_{1}^{-1} f(w)+z+J_{1}^{-1} J_{2} s, \\
M(w)+c=z+J_{1}^{-1} J_{2} s-J_{1}^{-1} \mathrm{f}(w), \\
\text { where } c=\gamma+J_{1}^{-1} J_{2} \eta-J_{1}^{-1} f(\delta), \\
f(w)=J_{1} z+J_{2} s-J_{1}(M(w)+c) .
\end{gathered}
$$

From (44), we obtain

$$
L(z)+J_{1} z+J_{2} s=J_{1} c+J_{1} M(w) .
$$

We decompose the solution into infinite series sum

$$
w=\sum_{i=0}^{\infty} w_{i}
$$

Equation (48) implies

$$
\begin{gathered}
L\left(\sum_{i=0}^{\infty} z_{i}\right)+J_{1}\left(\sum_{i=0}^{\infty} z_{i}\right)+J_{2}\left(\sum_{i=0}^{\infty} s_{i}\right)=J_{1} c+J_{1} M\left(\sum_{i=0}^{\infty} w_{i}\right) \\
L\left(\sum_{i=0}^{\infty} z_{i}\right)+J_{1}\left(\sum_{i=0}^{\infty} z_{i}\right)+J_{2}\left(\sum_{i=0}^{\infty} s_{i}\right) \\
=J_{1} c+J_{1}\left(M\left(w_{0}\right)+\left(M\left(w_{0}+w_{1}\right)-M\left(w_{0}\right)\right)\right. \\
+\left(M\left(w_{0}+w_{1}+w_{2}\right)\right. \\
\left.\left.-M\left(w_{0}+w_{1}\right)\right)+\cdots\right) \\
L\left(z_{0}\right)+J_{1} z_{0}+J_{2} s_{0}=J_{1} c
\end{gathered}
$$

$$
\begin{gathered}
L\left(z_{0}\right)+J_{1} z_{0}+J_{2} s_{0}=J_{1} \gamma+J_{2} \eta-f(\delta), \\
L\left(z_{0}\right)+J w_{0}=J \delta-f(\delta), \quad \text { where } J=\left.\frac{\partial f}{\partial w}\right|_{w=\delta}, \\
L\left(z_{1}\right)+J w_{1}=J_{1} M\left(w_{0}\right), \\
z_{n}^{\prime \prime}(x)+\frac{k}{x} z_{n}^{\prime}(x)+J_{0} z_{n}(x)=J_{0} y_{n}(x)-f\left(y_{n}(x)\right), \\
L\left(z_{2}\right)+J w_{2}=J_{1}\left(M\left(w_{0}+w_{1}\right)-M\left(w_{0}\right)\right), \\
\vdots \\
L\left(z_{n+1}\right)+J w_{n+1}=J_{1}\left(M\left(w_{0}+w_{1}+w_{2}+\cdots+w_{n}\right)\right. \\
\left.-M\left(w_{0}+w_{1}+w_{2}+\cdots+w_{n-1}\right)\right) .
\end{gathered}
$$

By adding (51) and (53), we get

$$
\begin{aligned}
L\left(z_{0}\right. & \left.+z_{1}+\cdots+z_{n+1}\right)+J\left(w_{0}+w_{1}+\cdots+w_{n+1}\right) \\
& =J_{1}\left(c+M\left(w_{0}+w_{1}+\cdots+w_{n}\right)\right) .
\end{aligned}
$$

We denote

$$
\begin{gathered}
w \approx W_{n+1}=w_{0}+w_{1}+\cdots+w_{n+1}, \\
z \approx Z_{n+1}=z_{0}+z_{1}+\cdots+z_{n+1} . \\
s \approx S_{n+1}=s_{0}+s_{1}+\cdots+s_{n+1} .
\end{gathered}
$$

From (54) and (46),

$$
\begin{gathered}
L\left(Z_{n+1}\right)+J W_{n+1}=J_{1}\left(c+M\left(W_{n}\right)\right), \\
L\left(Z_{n+1}\right)+J W_{n+1}=J_{1} Z_{n}+J_{2} S_{n}-f\left(W_{n}\right), \\
L\left(Z_{n+1}\right)+J W_{n+1}=J W_{n}-f\left(W_{n}\right) .
\end{gathered}
$$

After renaming the variables, we get the following iterative schemes.

Scheme $S W_{0}$. Consider

$$
L\left(z_{1}\right)+J w_{1}=J w_{0}-f\left(w_{0}\right), \quad \text { where } J=\left.\frac{\partial f}{\partial w}\right|_{w=w_{0}} .
$$

Scheme $S W_{1}$. Consider

$$
\begin{aligned}
& L\left(z_{1}\right)+J w_{1}=J w_{0}-f\left(w_{0}\right), \\
& L\left(w_{2}\right)+J w_{2}=J w_{1}-f\left(w_{1}\right) .
\end{aligned}
$$

Scheme $S W_{2}$. Consider

$$
\begin{aligned}
& L\left(z_{1}\right)+J w_{1}=J w_{0}-f\left(w_{0}\right), \\
& L\left(z_{2}\right)+J w_{2}=J w_{1}-f\left(w_{1}\right), \\
& L\left(z_{3}\right)+J w_{3}=J w_{2}-f\left(w_{2}\right) .
\end{aligned}
$$


TABLE 1: Convergence order and absolute error for Bratu problem for $\lambda=1$ and different values of $N$.

\begin{tabular}{|c|c|c|c|c|}
\hline \multirow{2}{*}{ Iter. } & \multicolumn{4}{|c|}{$N$} \\
\hline & 50 & 100 & 150 & 200 \\
\hline \multicolumn{5}{|l|}{$S_{0}$} \\
\hline 1 & 0.000764899 & 0.000765714 & 0.000765861 & 0.000765913 \\
\hline 2 & $3.22437 e-8$ & $3.228 e-8$ & $3.22866 e-8$ & $3.22889 e-8$ \\
\hline 3 & $5.67396 e-17$ & $5.68041 e-17$ & $5.68158 e-17$ & $5.68198 e-17$ \\
\hline 4 & $1.75415 e-34$ & $1.75614 e-34$ & $1.7565 e-34$ & $1.75663 e-34$ \\
\hline 5 & $7.71203 e-49$ & $1.67808 e-69$ & $1.67843 e-69$ & $1.67855 e-69$ \\
\hline \multirow{3}{*}{ Rate } & 2.03003 & 2.03014 & 2.03016 & 2.03017 \\
\hline & 2.00097 & 2.00098 & 2.00098 & 2.00098 \\
\hline & 2.00008 & 2.00008 & 2.00008 & 2.00008 \\
\hline \multicolumn{5}{|l|}{$S_{1}$} \\
\hline 1 & 0.000010196 & 0.0000102072 & 0.0000102092 & 0.0000102099 \\
\hline 2 & $6.3566 e-18$ & $6.36381 e-18$ & $6.36511 e-18$ & $6.36557 e-18$ \\
\hline 3 & $7.71203 e-49$ & $1.52735 e-54$ & $1.52766 e-54$ & $1.52777 e-54$ \\
\hline 4 & $7.71203 e-49$ & $1.14254 e-95$ & $3.04915 e-142$ & $2.11033 e-164$ \\
\hline \multirow{2}{*}{ Rate } & 3.02829 & 3.0284 & 3.02842 & 3.02842 \\
\hline & 2.53302 & 3.00035 & 3.00035 & 3.00035 \\
\hline \multicolumn{5}{|l|}{$S_{2}$} \\
\hline 1 & $1.35077 e-7$ & $1.35226 e-7$ & $1.35253 e-7$ & $1.35262 e-7$ \\
\hline 2 & $2.18092 e-31$ & $2.1834 e-31$ & $2.18384 e-31$ & $2.184 e-31$ \\
\hline 3 & $7.71203 e-49$ & $1.1425376 e-95$ & $1.46619 e-126$ & $1.46629 e-126$ \\
\hline Rate & 4.02691 & 4.02701 & 4.02702 & 4.02703 \\
\hline \multicolumn{5}{|l|}{$S_{3}$} \\
\hline 1 & $1.7892 e-9$ & $1.79116 e-9$ & $1.79152 e-9$ & $1.79164 e-9$ \\
\hline 2 & $1.79244 e-48$ & $1.31437 e-48$ & $1.31464 e-48$ & $1.31473 e-48$ \\
\hline 3 & $7.71203 e-49$ & $1.14254 e-95$ & $3.04915 e-142$ & $1.03327 e-188$ \\
\hline Rate & 5.00878 & 5.02624 & 5.02626 & 5.02627 \\
\hline
\end{tabular}

Scheme $S W_{n}$. Consider

$$
\begin{gathered}
L\left(z_{1}\right)+J w_{1}=J w_{0}-f\left(w_{0}\right), \\
L\left(z_{2}\right)+J w_{2}=J w_{1}-f\left(w_{1}\right), \\
L\left(z_{3}\right)+J w_{3}=J w_{2}-f\left(w_{2}\right), \\
\vdots \\
L\left(z_{n+1}\right)+J w_{n+1}=J w_{n}-f\left(w_{n}\right) .
\end{gathered}
$$

\section{Numerical Results and Rate of Convergence}

In all numerical experimentation, we use Chebyshev pseudospectral methods (for more details, see [13]). In order to show the rate of convergence, we require the definition of computational order of convergence, The computational order of convergence can be approximated by [28]

$$
\mathrm{COC} \approx \frac{\ln \left\|\left(y_{n+1}-\alpha\right)\left(y_{n}-\alpha\right)^{-1}\right\|_{\infty}}{\ln \left\|\left(y_{n}-\alpha\right)\left(y_{n-1}-\alpha\right)^{-1}\right\|_{\infty}},
$$

where $y_{n-1}, y_{n}$, and $y_{n+1}$ are successive iterations closer to the solution $\alpha(x)$ of boundary value problem and $\left\|v_{q}\right\|_{\infty}=$ $\max \left\{\left|v_{q}\left(x_{1}\right)\right|,\left|v_{q}\left(x_{2}\right)\right|, \ldots,\left|v_{q}\left(x_{N}\right)\right|\right\}$ such that $\left\{x_{1}, x_{2}, \ldots, x_{N}\right\}$ defines the partition of domain of the BVP. For BVPs with infinite domain, for instance, $[a, \infty),(-\infty, a]$, or $(-\infty, \infty)$, one could replace infinity by a suitable large number to make the domain compact. The iterative scheme $S_{0}$ for Bratu problem with boundary conditions is

$$
\begin{gathered}
y_{n+1}^{\prime \prime}(x)+\lambda e^{y_{n}(x)} y_{n+1}(x)=\lambda e^{y_{n}(x)} y_{n}(x)-\lambda e^{y_{n}(x)}, \\
y_{n+1}(0)=y_{n+1}(1)=0,
\end{gathered}
$$

and $y_{0}(x)=x(1-x)$ is an initial guess for (62). Similarly, we can obtain the scheme $S_{1}$ as follows:

$$
\begin{gathered}
z_{n}^{\prime \prime}(x)+\lambda e^{y_{n}(x)} z_{n}(x)=\lambda e^{y_{n}(x)} y_{n}(x)-\lambda e^{y_{n}(x)}, \\
y_{n+1}^{\prime \prime}(x)+\lambda e^{y_{n}(x)} y_{n+1}(x)=\lambda e^{y_{n}(x)} z_{n}(x)-\lambda e^{z_{n}(x)} .
\end{gathered}
$$


TABLE 2: Convergence order for Bratu problem for $\lambda=3$ and different values of $N$.

\begin{tabular}{|c|c|c|c|}
\hline \multirow{2}{*}{ Iter. } & \multicolumn{3}{|c|}{$N$} \\
\hline & 50 & 100 & 150 \\
\hline \multicolumn{4}{|l|}{$\overline{S_{0}}$} \\
\hline 1 & 0.0458144 & 0.045864 & 0.045873 \\
\hline 2 & 0.000954118 & 0.000955184 & 0.000955377 \\
\hline 3 & $4.40809 e-7$ & $4.41304 e-7$ & $4.41393 e-7$ \\
\hline 4 & $9.41641 e-14$ & $9.42698 e-14$ & $9.4289 e-14$ \\
\hline 5 & $4.29662 e-27$ & $4.30146 e-27$ & $4.30234 e-27$ \\
\hline \multirow{3}{*}{ Rate } & 1.80854 & 1.80867 & 1.80869 \\
\hline & 1.98368 & 1.98369 & 1.98369 \\
\hline & 1.9999 & 1.9999 & 1.9999 \\
\hline \multicolumn{4}{|l|}{$\begin{array}{l}S_{1} \\
\end{array}$} \\
\hline 1 & 0.0104901 & 0.0105016 & 0.0105037 \\
\hline 2 & $5.27186 e-7$ & $5.27777 e-7$ & $5.27884 e-7$ \\
\hline 3 & $6.88292 e-20$ & $6.89065 e-20$ & $6.89205 e-20$ \\
\hline 4 & $1.80799 e-32$ & $1.53308 e-58$ & $1.503335 e-58$ \\
\hline \multirow{2}{*}{ Rate } & 2.73823 & 2.73837 & 2.73839 \\
\hline & 2.99715 & 2.99716 & 2.99716 \\
\hline \multicolumn{4}{|l|}{$S_{2}$} \\
\hline 1 & 0.00253291 & 0.0025357 & 0.00253621 \\
\hline 2 & $1.86441 e-11$ & $1.86651 e-11$ & $1.86688 e-11$ \\
\hline 3 & $1.80799 e-32$ & $5.50708 e-44$ & $5.5082 e-44$ \\
\hline Rate & 3.71869 & 3.71882 & 3.71885 \\
\hline \multicolumn{4}{|l|}{$S_{3}$} \\
\hline 1 & 0.000618859 & 0.000619541 & 0.000619664 \\
\hline 2 & $4.0232 e-17$ & $4.02771 e-17$ & $4.02853 e-17$ \\
\hline 3 & $1.80799 e-32$ & $4.68 e-63$ & $4.65698 e-83$ \\
\hline Rate & 4.71115 & 4.71128 & 4.7113 \\
\hline
\end{tabular}

TABLE 3: Iterative convergence for different schemes for the critical value of $\lambda_{c}$ for Bratu problem, $N=200$.

\begin{tabular}{cccc}
\hline & & Iter. & $S_{2}$ \\
\hline Critical $\lambda_{c}$ & & $S_{1}$ & \\
0 & 2.94303552937154 & 2.94303552937154 & 2.94303552937154 \\
5 & 3.63644753861909 & 3.51376131743441 & 3.95335426360815 \\
10 & 3.51383579457353 & 3.51383064747221 & 3.51383072002551 \\
15 & 3.5138308350082 & 3.5138307190047 & 3.51383071919088 \\
Absolute errors & & & \\
0 & 0.570795189753462 & 0.570795189753462 & 0.570795189753462 \\
5 & 0.122616819494092 & $6.94016905877781 e-005$ & 0.439523544483147 \\
10 & $5.07544853212138 e-006$ & $7.16527859268012 e-008$ & $9.00510332968452 e-010$ \\
15 & $1.15883229678815 e-007$ & $1.20208731857474 e-010$ & $6.58797461028371 e-011$ \\
\hline
\end{tabular}

The $S_{2}$ and $S_{3}$ schemes are

$$
\begin{gathered}
z_{n}^{\prime \prime}(x)+\lambda e^{y_{n}(x)} z_{n}(x)=\lambda e^{y_{n}(x)} y_{n}(x)-\lambda e^{y_{n}(x)}, \\
w_{n}^{\prime \prime}(x)+\lambda e^{y_{n}(x)} w_{n}(x)=\lambda e^{y_{n}(x)} z_{n}(x)-\lambda e^{z_{n}(x)}, \\
y_{n+1}^{\prime \prime}(x)+\lambda e^{y_{n}(x)} y_{n+1}(x)=\lambda e^{y_{n}(x)} w_{n}(x)-\lambda e^{w_{n}(x)},
\end{gathered}
$$

$$
\begin{gathered}
z_{n}^{\prime \prime}(x)+\lambda e^{y_{n}(x)} z_{n}(x)=\lambda e^{y_{n}(x)} y_{n}(x)-\lambda e^{y_{n}(x)}, \\
w_{n}^{\prime \prime}(x)+\lambda e^{y_{n}(x)} w_{n}(x)=\lambda e^{y_{n}(x)} z_{n}(x)-\lambda e^{z_{n}(x)}, \\
p_{n}^{\prime \prime}(x)+\lambda e^{y_{n}(x)} p_{n}(x)=\lambda e^{y_{n}(x)} w_{n}(x)-\lambda e^{w_{n}(x)}, \\
y_{n+1}^{\prime \prime}(x)+\lambda e^{y_{n}(x)} y_{n+1}(x)=\lambda e^{y_{n}(x)} p_{n}(x)-\lambda e^{p_{n}(x)},
\end{gathered}
$$


TABLE 4: Convergence order for Frank-Kamenetzkii problem for $k=1, \lambda=1$ and different values of $N$.

\begin{tabular}{|c|c|c|c|}
\hline \multirow{2}{*}{ Iter. } & \multicolumn{3}{|c|}{$N$} \\
\hline & 50 & 100 & 150 \\
\hline \multicolumn{4}{|c|}{$S_{0}$} \\
\hline 1 & 0.114557 & 0.114557 & 0.114557 \\
\hline 2 & 0.00125407 & 0.00125407 & 0.00125407 \\
\hline 3 & $1.58064 e-7$ & $1.58064 e-7$ & $1.58064 e-7$ \\
\hline 4 & $2.48121 e-15$ & $2.48121 e-15$ & $2.48121 e-15$ \\
\hline 5 & $6.09322 e-31$ & $6.09322 e-31$ & $6.09322 e-31$ \\
\hline \multirow{3}{*}{ Rate } & 2.52799 & 2.52799 & 2.52799 \\
\hline & 1.98883 & 1.98883 & 1.98883 \\
\hline & 2.00133 & 2.00133 & 2.00133 \\
\hline \multicolumn{4}{|l|}{$S_{1}$} \\
\hline 1 & 0.0329952 & 0.0329952 & 0.0329952 \\
\hline 2 & $7.69119 e-7$ & $7.69119 e-7$ & $7.69119 e-7$ \\
\hline 3 & $8.94424 e-21$ & $8.94424 e-21$ & $8.94424 e-21$ \\
\hline 4 & $9.38343 e-52$ & $1.39988 e-62$ & $1.39988 e-62$ \\
\hline \multirow{2}{*}{ Rate } & 3.51966 & 3.51966 & 3.51966 \\
\hline & 3.008 & 3.008 & 3.008 \\
\hline \multicolumn{4}{|l|}{$S_{2}$} \\
\hline 1 & 0.00877215 & 0.00877215 & 0.00877215 \\
\hline 2 & $2.38344 e-11$ & $2.38344 e-11$ & $2.38344 e-11$ \\
\hline 3 & $1.25899 e-45$ & $1.25899 e-45$ & $1.25899 e-45$ \\
\hline Rate & 4.52861 & 4.52861 & 4.52861 \\
\hline \multicolumn{4}{|l|}{$S_{3}$} \\
\hline 1 & 0.00237755 & 0.00237755 & 0.00237755 \\
\hline 2 & $6.27393 e-17$ & $6.27393 e-17$ & $6.27393 e-17$ \\
\hline 3 & $9.38343 e-52$ & $7.50684 e-85$ & $7.50684 e-85$ \\
\hline Rate & 5.52316 & 5.52316 & 5.52316 \\
\hline
\end{tabular}

TABLE 5: Iterative convergence for different schemes for the critical value of $\lambda_{c}$ for Frank-Kamenetzkii problem, $N=200$.

\begin{tabular}{cccc}
\hline & & Iter. & \\
& $S_{0}$ & $S_{1}$ & \\
\hline Critical $\lambda_{c}$ & & & \\
0 & 1.96202368624769 & 1.96202368624769 & 1.96202368624769 \\
1 & 2.00031964646299 & 1.99933495330998 & 1.99987632667614 \\
2 & 2.00025140688174 & 2.00000007821613 & 1.99999999897116 \\
3 & 2.00000003077048 & 2.00000000000462 & 1.99999999999956 \\
4 & 2.00000001017936 & 1.99999999999926 & 1.99999999999949 \\
Absolute errors & & & \\
0 & 0.0379763137523081 & 0.0379763137523081 & 0.0379763137523081 \\
1 & 0.000319646462989098 & 0.000665046690023985 & 0.000123673323863427 \\
2 & 0.000251406881741634 & $7.82161291290606 e-008$ & $1.02883590535896 e-009$ \\
4 & $3.07704830504463 e-008$ & $4.6216364069096 e-012$ & $4.42312853010662 e-013$ \\
& $1.0179361797924 e-008$ & $7.41184891239755 e-013$ & $5.14255305006373 e-013$ \\
\hline
\end{tabular}


TABLE 6: Iterative convergence for different schemes for the critical value of $\alpha_{c}$ for Frank problem, $N=200$.

\begin{tabular}{lccc}
\hline & & Iter. & \\
& $S_{0}$ & $S_{1}$ & $S_{2}$ \\
\hline Critical $\alpha_{c}$ & & & \\
0 & 1.33333333333333 & 1.33333333333333 & 1.333333333333333 \\
1 & 1.37022694167246 & 1.38416475416079 & 1.38597531980146 \\
2 & 1.38643019839731 & 1.38630914733076 & 1.38629436351113 \\
3 & 1.3864007758502 & 1.38629428714795 & 1.38629438822645 \\
4 & 1.38629414907138 & 1.38629433591242 & 1.38629439729082 \\
Absolute errors & & & 0.0529610277866668 \\
0 & 0.0529610277866668 & 0.0529610277866668 & 0.00031904131854454 \\
1 & 0.0160674194475423 & 0.0021296069592109 & $2.39112551980725 e-009$ \\
3 & 0.000135837277305928 & $1.47862107602315 e-005$ & $2.71064524159925 e-008$ \\
4 & 0.000106414730195015 & $7.3972053149518 e-008$ & $3.61708230034452 e-008$ \\
\hline
\end{tabular}

respectively. Tables 1 and 2 show the infinity norms of error and rates of convergence for (62), (63), (64), and (65) for $\lambda=$ $1, \lambda=3$ and $N$ runs over 50,100, 150, and 200. We denote $f(y)=\lambda e^{y(x)}$ and $f_{y}(y)=\partial f(y) / \partial y=\lambda e^{y(x)}$ and $J_{0}=$ $f_{y}\left(y_{0}(x)\right)$. The construction of $S_{3}$ scheme for (2) is given below and others are similar. Consider

$$
\begin{gathered}
z_{n}^{\prime \prime}(x)+\frac{k}{x} z_{n}^{\prime}(x)+J_{0} z_{n}(x)=J_{0} y_{n}(x)-f\left(y_{n}(x)\right), \\
w_{n}^{\prime \prime}(x)+\frac{k}{x} w_{n}^{\prime}(x)+J_{0} w_{n}(x)=J_{0} z_{n}(x)-f\left(z_{n}(x)\right), \\
p_{n}^{\prime \prime}(x)+\frac{k}{x} p_{n}^{\prime}(x)+J_{0} p_{n}(x)=J_{0} w_{n}(x)-f\left(w_{n}(x)\right), \\
y_{n+1}^{\prime \prime}(x)+\frac{k}{x} y_{n+1}^{\prime}(x)+J_{0} y_{n+1}(x) \\
=J_{0} p_{n}(x)-f\left(p_{n}(x)\right), \\
y_{n+1}^{\prime}(0)=y_{n+1}(1)=0 .
\end{gathered}
$$

The initial guess $y_{0}(x)=1-x^{2}$ for Frank-Kamenetzkii problem is used to start the proposed schemes. The infinity norms of error and rates of convergence for Frank-Kamenetzkii problem $(\lambda=1, k=1)$ are depicted in Table 4 . In order to plot bifurcation diagram for the one-dimensional Bratu problem and the Frank-Kamenetzkii problem, we rewrite the both problems as follows:

$$
\begin{gathered}
u^{\prime \prime}(x)+\lambda e^{u(x)}=0, \quad u(0)=u(1)=0, \\
u^{\prime \prime}(x)+\frac{k}{x} u^{\prime}(x)+\eta e^{u(x)}=0, \quad u^{\prime}(0)=u(1)=0 .
\end{gathered}
$$

If $\lambda<\lambda_{c}(=3.51383071912516), \lambda=\lambda_{c}$, and $\lambda>\lambda_{c}$, then the Bratu problem has two solutions, unique solution and no solution, respectively, and similarly, for the FrankKamenetzkii problem statement is valid if $k=1$ and $\eta_{c}=2$. We define B1-problem and FK1-problem

$$
\begin{gathered}
u^{\prime \prime}(x)+\lambda e^{u(x)}=0, \\
u(0)=0, \quad u^{\prime}(0)=\alpha, \quad u(1)=0, \\
u^{\prime \prime}(x)+\frac{k}{x} u^{\prime}(x)+\lambda e^{u(x)}=0, \\
u^{\prime}(0)=0, \quad u(0)=\alpha, \quad u(1)=0,
\end{gathered}
$$

respectively. The iterative forms of (68) and (69) are

$$
\begin{gathered}
u_{n+1}^{\prime \prime}(x)+\lambda_{n} e^{u_{n}(x)} u_{n+1}(x)+e^{u_{n}(x)} \lambda_{n+1}=\lambda_{n} e^{u_{n}(x)} u_{n}(x), \\
u(0)=0, \quad u^{\prime}(0)=\alpha_{1}, \quad u(1)=0, \\
u_{n+1}^{\prime \prime}(x)+\frac{k}{x} u_{n+1}^{\prime}(x)+\eta_{n} e^{u_{n}(x)} u_{n+1}(x) \\
+e^{u_{n}(x)} \eta_{\mathrm{n}+1}=\eta_{n} e^{u_{n}(x)} u_{n}(x), \\
u(0)=\alpha_{2}, \quad u^{\prime}(0)=0, \quad u(1)=0 .
\end{gathered}
$$

The bifurcation diagrams are shown in Figures 1 and 2. The calculation of critical parameters for the B1-problem and FK1problem is performed by using proposed system of equation in [13] and Tables 2, 5, and 6 show numerical results of different iterative schemes for B1-problem and FK1-problem.

The authors of [13] are pioneer to talk about higherorder iterative quasilinearization method (QLM). Tables 1, 2, and 4 confirm the orders of convergence of their respective iterative schemes for the different values of parameters under a different range of grid points for Chebyshev pseudospectral method. We make all the calculation for Tables 1, 2, and 4 in Mathematica (MinPrecision =200). The scheme-0 in [13] and $S_{0}$ in this paper are the same because both are QLM and the calculated results should be same but unfortunately this is not the case. For the QLM, the infinity norm of 


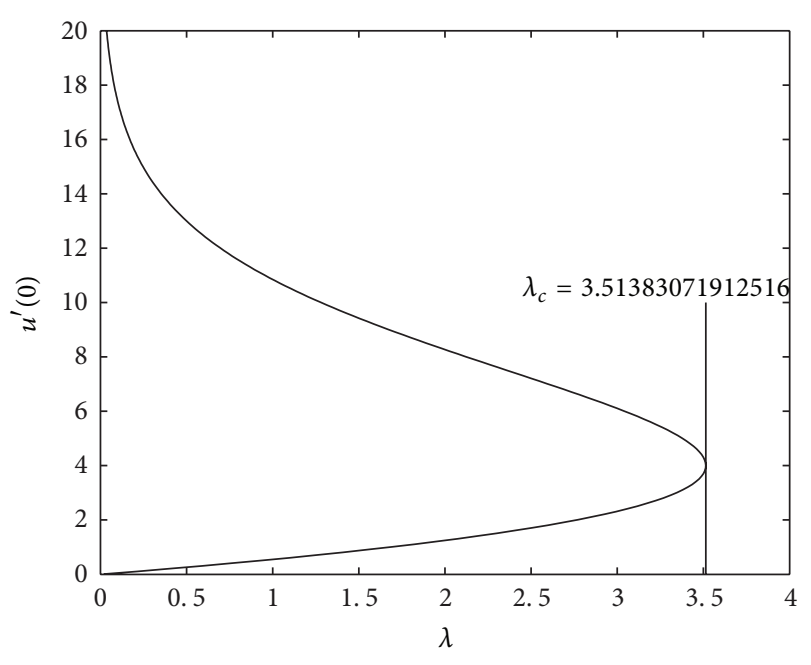

FIgURE 1: Bifurcation diagram for Bratu problem (7).

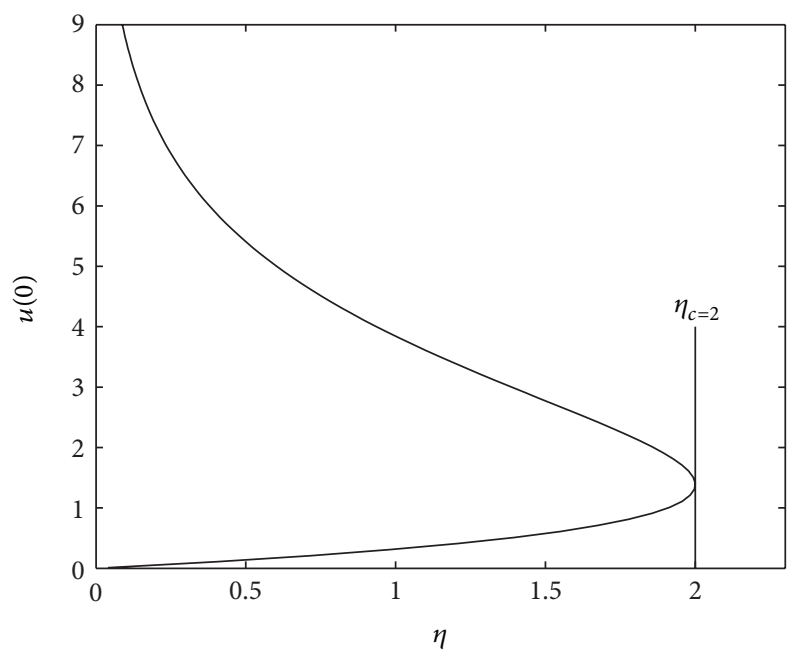

FIGURE 2: Bifurcation diagram for Frank-Kamenetzkii problem (8).

error for Bratu problem is (Scheme-0, $N=50,6.66 e-49$ ), (Scheme-2, $N=100,6.34 e-49)$, (Scheme-2, $N=150$, 6.34e-49), and (Scheme-3, $N=200,6.34 e-49)$ in Table 1 [13] and in this article, in Table $1\left(S_{0}, N=50,7.7 e-49\right)$, $\left(S_{1}, N=100,1.67 e-69\right),\left(S_{2}, N=150,1.67 e-69\right),\left(S_{3}\right.$, $N=200,1.67 e-69)$ and in other cases, our results show better reduction in error as compared to [13] for Table 1 as well as for Table 2 for Bratu problem. For the case of Frank-Kamenetzkii problem, Table 4 results are comparable with the reported results in Table 4 [13] and especially for QLM case almost results are the same. The Table 3 shows the computation of critical parameter $\lambda_{c}$ for Bratu problem, and again the results produced in this article and in [13] for QLM are not the same which in fact should be the same. The results presented by other authors are surprisingly superior for QLM and for other schemes. Notice that we use Matlab to compute critical parameters. Tables 5 and 6 of [13] show better performance and our results are comparable with them. It is noticed that in [13], the constructed matrix systems (33) and
(34) for the Bratu and the Frank-Kamenetzkii problems have not properly implemented for all boundary conditions. It is also noted that in some of the cases, if we increase the grid points by keeping the same scheme, there is an improvement in the accuracy of the calculated results, but in some cases this is not valid rule. It is also very clear from Tables 1,2 , and 4, that we ensure the convergence order which was the claim.

\section{Conclusions}

An efficient method is presented in this article to get arbitrary higher-order iterative schemes. The construction of iterative schemes is very simple and straightforward. In [13], authors used the idea to decompose the nonlinear operator by using Adomain decomposition method (ADM) which requires the calculation of Adomain polynomials and in a result, the computational cost will be high. In our case, there is no need to calculate any extra polynomial in order to enhance the order of convergence of an iterative method and both methods require only one inversion of Jacobian. Our presented method is computationally efficient because it does not require to construct any Adomain polynomial and hence implementation is also simple to achieve the same order of convergence. We think that the results presented in [13] and in this article should be the same for the case of QLM, but it is not the case and we do not know the implementation of the iterative schemes in [13].

\section{Acknowledgment}

This work was funded by the Deanship of Scientific Research (DSR), King Abdulaziz University, Jeddah, under Grant No. (130-014-D1434). The authors, therefore, acknowledge with thanks DSR technical and financial support.

\section{References}

[1] H. T. Kung and J. F. Traub, "Optimal order of one-point and multipoint iteration," Journal of the Association for Computing Machinery, vol. 21, pp. 643-651, 1974.

[2] S. K. Khattri, M. A. Noor, and E. Al-Said, "Unifying fourthorder family of iterative methods," Applied Mathematics Letters, vol. 24, no. 8, pp. 1295-1300, 2011.

[3] R. F. King, "A family of fourth order methods for nonlinear equations," SIAM Journal on Numerical Analysis, vol. 10, pp. 876-879, 1973.

[4] P. Sargolzaei and F. Soleymani, "Accurate fourteenth-order methods for solving nonlinear equations," Numerical Algorithms, vol. 58, no. 4, pp. 513-527, 2011.

[5] J. E. Traub, Iterative Methods For the Solution of Equations, Chelsea Publishing company, New york, NY, USA, 1976.

[6] Y. H. Geum and Y. I. Kim, "A multi-parameter family of threestep eighth-order iterative methods locating a simple root," Applied Mathematics and Computation, vol. 215, no. 9, pp. 3375$3382,2010$.

[7] A. M. Ostrowski, Solution of Equations and System If Equations, Academic Press, New York, NY, USA, 1960. 
[8] R. Krivec and V. B. Mandelzweig, "Numerical investigation of quasilinearization method in quantum mechanics," Computer Physics Communications, vol. 138, no. 1, pp. 69-79.

[9] V. B. Mandelzweig, "Quasilinearization method and its verification on exactly solvable models in quantum mechanics," Journal of Mathematical Physics, vol. 40, no. 12, pp. 6266-6291, 1999.

[10] V. B. Mandelzweig and F. Tabakin, "Quasilinearization approach to nonlinear problems in physics with application to nonlinear ODEs," Computer Physics Communications, vol. 141, no. 2, pp. 268-281, 2001.

[11] V. B. Mandelzweig, "Quasilinearization method: nonperturbative approach to physical problems," Physics of Atomic Nuclei, vol. 68, no. 7, pp. 1227-1258, 2005.

[12] R. E. Bellman and R. E. Kalaba, Quasilinearization and Nonlinear Boundary-Value Problems, Elsevier, New York, NY, USA, 1965.

[13] S. S. Motsa and P. Sibanda, "Some modification of the quasilinearization method with higher-order convergence for solving nonlinear BVPs," Numerical Algorithms, vol. 63, no. 3, pp. 399417, 2013.

[14] G. Adomian, "A review of the decomposition method and some recent results for nonlinear equations," Mathematical and Computer Modelling, vol. 13, no. 7, pp. 17-43, 1990.

[15] V. Daftardar-Gejji and H. Jafari, "An iterative method for solving nonlinear functional equations," Journal of Mathematical Analysis and Applications, vol. 316, no. 2, pp. 753-763, 2006.

[16] P. Amore and F. M. Fernndez, "The virial theorem for nonlinear problems," 2009, arXiv: 0904.3858v2.

[17] J. P. Boyd, "Chebyshev polynomial expansions for simultaneous approximation of two branches of a function with application to the one-dimensional Bratu equation," Applied Mathematics and Computation, vol. 143, no. 2-3, pp. 189-200, 2003.

[18] J. P. Boyd, "One-point pseudospectral collocation for the onedimensional Bratu equation," Applied Mathematics and Computation, vol. 217, no. 12, pp. 5553-5565, 2011.

[19] T. F. C. Chan and H. B. Keller, "Arc-length continuation and multigrid techniques for nonlinear elliptic eigenvalue problems," Society for Industrial and Applied Mathematics, vol. 3, no. 2, pp. 173-194, 1982.

[20] I. H. Hassan and V. S. Ert, "Applying differential transformation method to the one-dimensional planar Bratu problem," International Journal of Contemporary Mathematical, vol. 2, no. 30, pp. 1493-1504, 2007.

[21] O. D. Makinde and E. Osalusi, "Exothermic explosions in symmetric geometriesan exploitation of perturbation technique," Romanian Journal of Physics, vol. 50.

[22] O. D. Makinde and E. Osalusi, "Exothermic explosions in symmetric geometriesan exploitation of perturbation technique," Romanian Journal of Physics, vol. 50, pp. 621-625, 2005.

[23] A.-M. Wazwaz, "Adomian decomposition method for a reliable treatment of the Bratu-type equations," Applied Mathematics and Computation, vol. 166, no. 3, pp. 652-663, 2005.

[24] D. A. Frank-Kamenetzkii, Diffusion and Heat Transfer in Chemical Kinetics, Plenum Press, New York, NY, USA, 1969.

[25] C. Harley and E. Momoniat, "Efficient boundary value problem solution for a Lane-Emden equation," Mathematical \& Computational Applications, vol. 15, no. 4, pp. 613-620, 2010.

[26] M. Kubcek and V. Hlavack, Numerical Solution of Nonlinear Boundary Value Problems With Applications, Prentice-Hall, Englewood Cliffs, New Jersey, 1983.
[27] M. A. Z. Raja and S.-U.-I. Ahmad, "Numerical treatment for solving one-dimensional Bratu problem using neural networks," Neural Computing and Applications, vol. 2012.

[28] S. Weerakoon and T. G. I. Fernando, "A variant of Newton's method with accelerated third-order convergence," Applied Mathematics Letters, vol. 13, no. 8, pp. 87-93, 2000. 


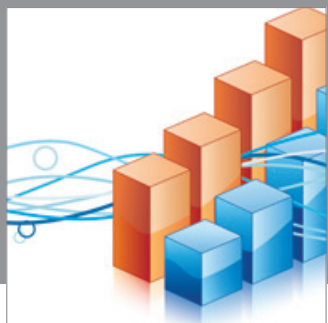

Advances in

Operations Research

mansans

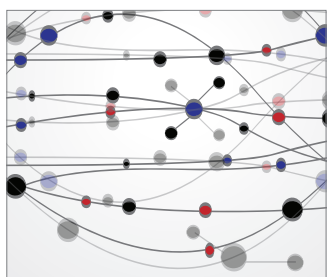

The Scientific World Journal
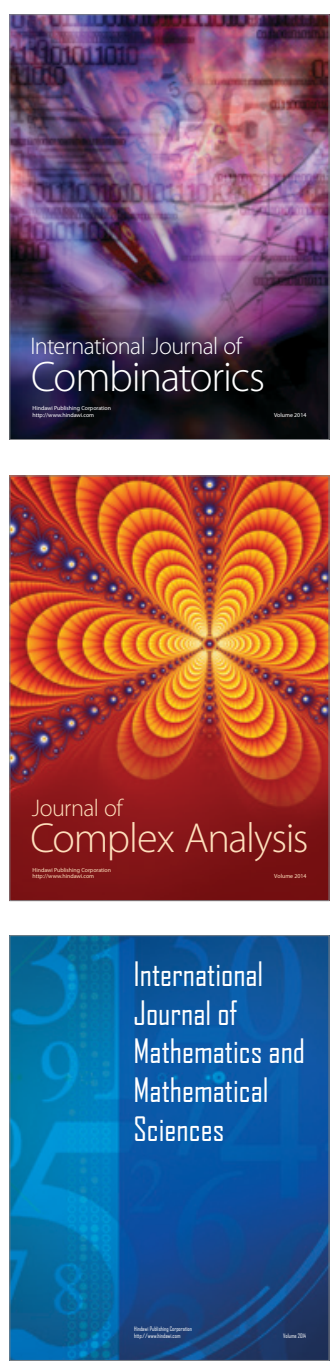
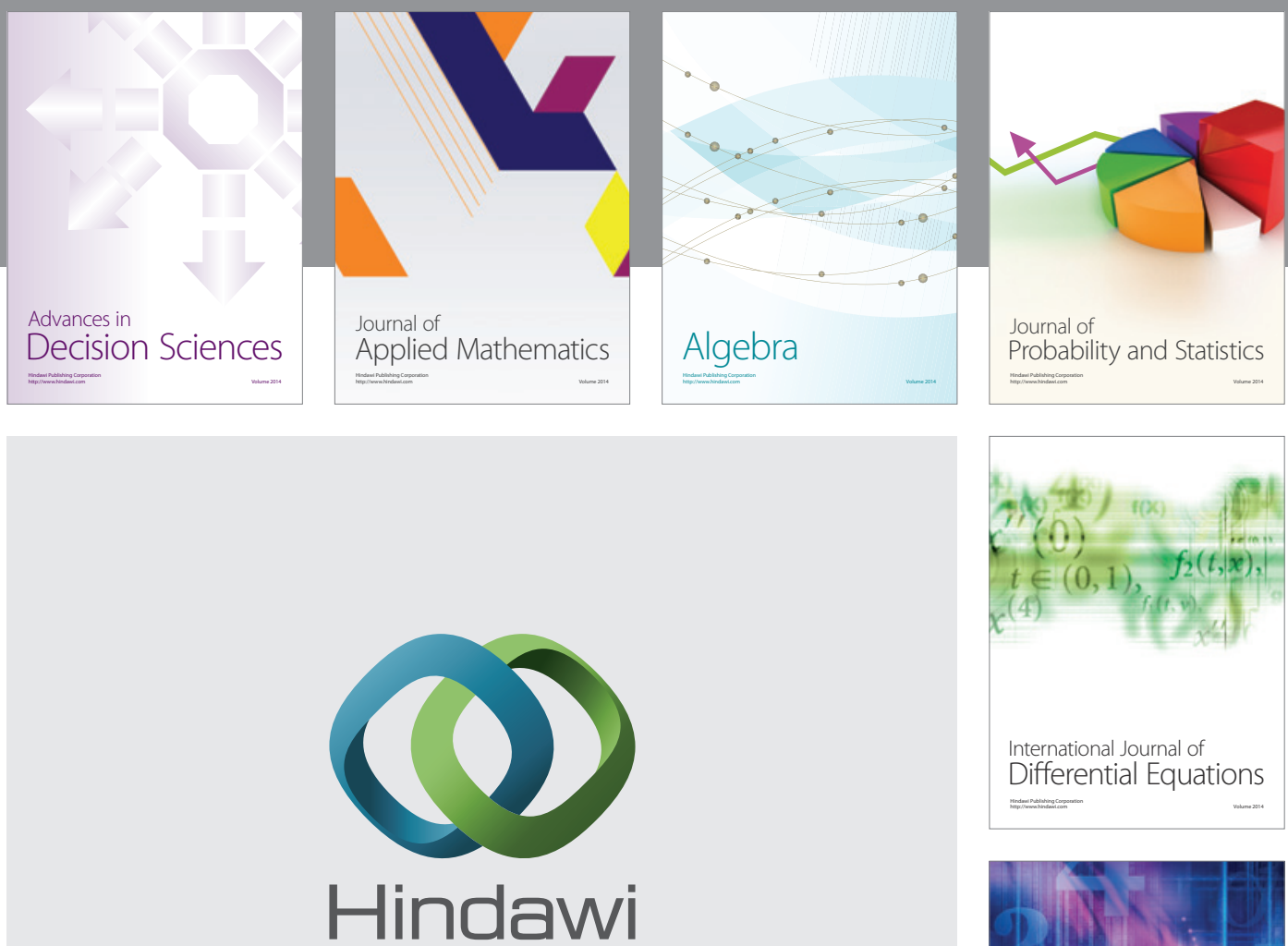

Submit your manuscripts at http://www.hindawi.com
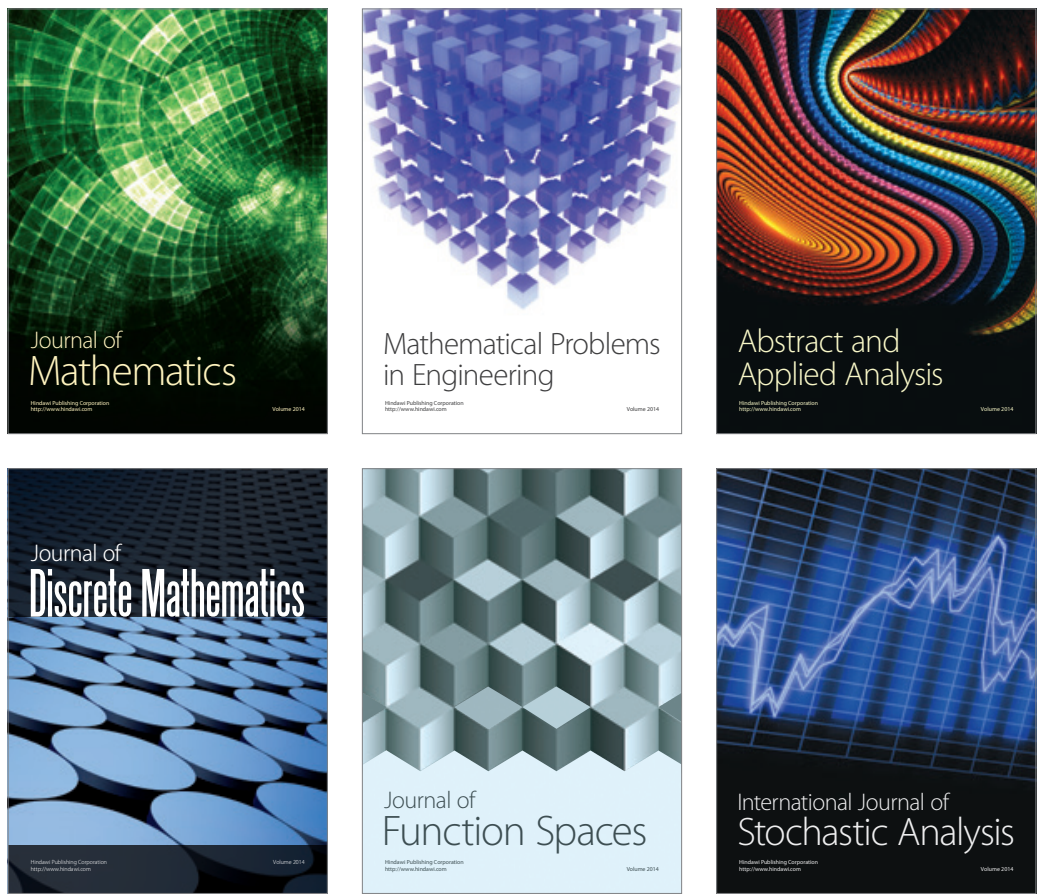

Journal of

Function Spaces

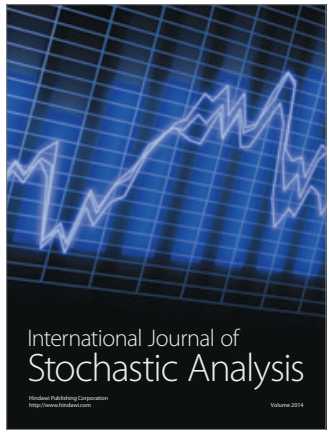

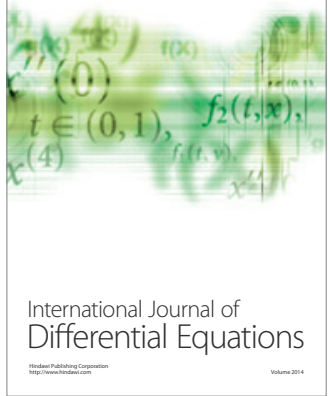
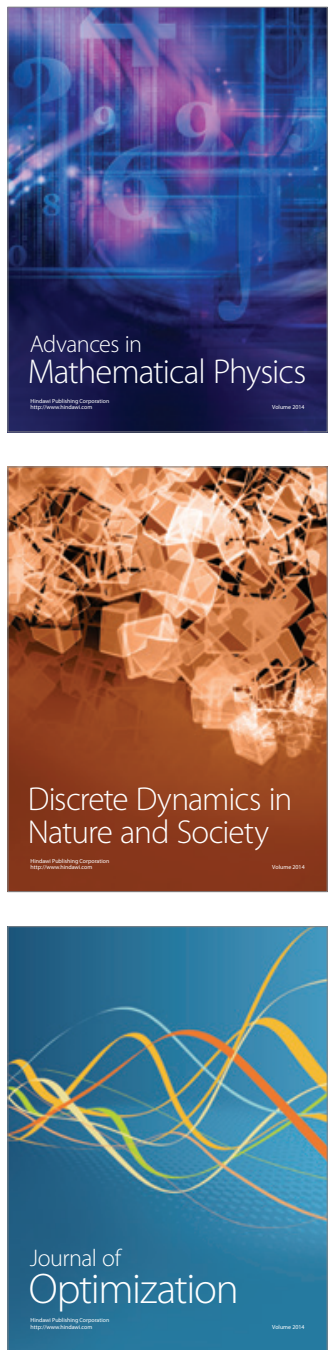